\title{
\& Research Square

\section{Audiovisual Teleconsultation For Patients With Epilepsy In Primary Care In Rural Germany: A Pilot Study On Feasibility And Acceptance}

Gregor Feldmeier ( $\nabla$ gregor.feldmeier@med.uni-rostock.de)

Rostock University Medical Center https://orcid.org/0000-0001-7420-5752

Christin Löffler

Rostock University Medical Center

Attila Altiner

Rostock University Medical Center

Anja Wollny

Rostock University Medical Center

\section{Felix von Podewils}

University Medicine Greifswald

Manuela Ritzke

Rostock University Medical Center

\section{Research}

Keywords: teleconsultation, remote consultation, telemedicine, distance counselling, e-therapy, e-health, general practice, family practice, general practitioner, epilepsy, neurologist

Posted Date: December 28th, 2021

DOI: https://doi.org/10.21203/rs.3.rs-1173135/v1

License: (c) (i) This work is licensed under a Creative Commons Attribution 4.0 International License.

Read Full License 


\section{Abstract}

Background: In rural areas, epilepsy patients have limited access to specialised care. They usually have to put up with travel and waiting times of several hours. In addition, often the communication between general practitioners (GP) and specialized epileptologists about diagnosis and treatment is complicated by the high workload on both sides and the different prioritization of treatment goals. Therefore, the aim of this study was to investigate the feasibility of an interprofessional audio-visual patient-doctor teleconsultation and its acceptance in clinical practice in patients with epilepsy in a rural region in Germany.

Method: Ten patients participated in telemedicine consultations in their GP practice. The practice was located in a sparsely populated region of Mecklenburg- Western Pomerania and equipped with technical equipment specifically procured for project-related purposes. An explorative qualitative interview was conducted with all participants. We based this paper on the consolidated criteria for reporting qualitative research (COREQ).

Results: Despite initial uncertainties on the part of the patients regarding the consultation setting, all participants found the teleconsultation helpful. Some patients were initially intimidated and felt slightly overwhelmed by the attention provided and the technology used (multiple HD cameras, large highresolution screens). However, during the consultation they felt supported by their GP and were satisfied that their needs were addressed in a satisfactory and timely manner. The hardware used was not felt to be a nuisance or to interfere with the conversation between doctor and patient. Patients also appreciated the time saved and the organisational convenience compared to a visit to a university outpatient clinic. Most consultations led to therapeutic consequences. Some patients seemed to benefit particularly, e.g. those who needed a change in their medication.

Conclusion: This pilot study provides first evidence that teleconsultations between patients, specialists and GPs are possible in rural areas. Interprofessional collaboration between GPs and epileptologists can improve the care of patients with epilepsy. Further research should investigate the effectiveness and efficiency of interprofessional telemedicine consultations for epilepsy and other conditions.

\section{Key Messages Regarding Feasibility}

1) What uncertainties existed regarding the feasibility? It was unclear to what extent patients would accept the new form of consultation with simultaneous meeting of patient, GP and specialist. 2) What are the key feasibility findings? The pilot study showed that patients quickly got used to the new situation of teleconsultation and were satisfied with the consultation outcome. The consultation model is suitable both for an initial contact with the epileptologist when epilepsy is suspected and for specialist follow-up care. 3) What are the implications of the feasibility findings for the design of the main study? For the design of the main study, a uniform appointment management as well as a documentation and billing system should be established. 


\section{Background}

Optimal health care for patients with epilepsy is a global challenge due to the hidden nature of this disease. [1] Disparities in treatment are described within countries, with a greater treatment gap in rural areas than in urban areas. This is attributed to a combination of inadequate access to services, stigma and negative beliefs and toward epilepsy, and low health literacy. [2]

In addition, treatment strategies appear to be different in rural areas. For instance, in rural Germany, drugs such as primidone and carbamazepine are prescribed more frequently, whereas prescribing of levetiracetam is more common in urban areas of the country. This is possibly due to the lack of experts in rural areas. [3] In addition to different treatment strategies and limited access to specialist consultations, travel time for patients who tend to be immobile and costs for visiting a specialist play an increasingly important role. With regard to climate change, it is important to reduce unnecessary journeys.

This is where telemedicine can make a special contribution. In their systematic review Wootton et al. use the example of tele-dermatology to show how many visits to the doctor can be avoided. The average percentage of avoided trips reported in the 12 store-and-forward studies (this means the collection, closed forwarding to experts and subsequent evaluation of patient data) was $43 \%$. In the 7 real-time studies and in a single study using a hybrid technique, journeys could be avoided for $70 \%$ of the patients. [4]

The travel times of patients for a visit to a neurologist in rural regions of Germany are given with a mean departure time of $42 \mathrm{~min}$ by car. In our patient population with epilepsy, there is also the factor that patients are unable to drive due to medication. In addition to distance, transport accessibility plays an important role. Since only about 62 percent of insured patients reported using a car for transportation, using public transportation increases the time for other patients [5]. Another study compared the accessibility of the general practitioner (GP) by public transport in a rural region of Germany with that of three exemplary specialists. A round trip to the GP averages 99 minutes, and, for example, to the urologists 160 minutes. As many as 6.5 percent of the inhabitants had no access to specialised internists by public transport, which should also apply to neurologists [6].

Furthermore, considering the demographic change and the increase of elderly patients, disease patterns as well as specialist care are going to change. For example, age related degenerative brain diseases such as Parkinson's and dementia [7] have already increased and will continue to do so in the future.

The pilot study aims at the feasibility and acceptance of an interprofessional audiovisual doctor-patient teleconsultation for epilepsy patients in rural regions of Germany. Possible indications for the improvement of the cooperation between GPs and specialised neurologists in structurally weak regions will be addressed. Research interest is further directed towards investigating the acceptance of innovative patient-centred care such as telemedicine counselling for selected neurological diseases such as epilepsy.

\section{Methods}




\section{Design}

For the realisation of a specialised telemedical consultation with a neurologist in a family practice, the technical requirements were first implemented and tested. Subsequently, we developed a concept for audiovisual GP-specialist-patient teleconsultations and implemented it with 10 patients. To investigate feasibility and acceptability, we chose an exploratory qualitative study design, as this allowed us to show both feasibility and possible barriers of the telemedicine service. To analyse experiences of all involved actors we interviewed the GP, the neurologist, and five of the ten participating patients. In reporting this study, we used the consolidated criteria for reporting qualitative research (COREQ) [8].

\section{Setting}

We conducted the pilot study in Mecklenburg-Western Pomerania, a predominantly rural region of Northeastern Germany. In a nationwide comparison it is characterised by a high poverty risk rate [9] and underserved regions for medical services or regions at risk of medical undersupply [10]. The selected patients took part in a teleconsultation with their GP as well as a specialist from an epilepsy outpatient clinic at the Epilepsy Centre of the Greifswald University Medical Centre. As a tertiary epilepsy centre, this is responsible for a population of more than 500,000 inhabitants.

\section{The pilot study}

Patients with a confirmed or suspected diagnosis of epilepsy participated in the pilot study. Patients who would normally have received a referral to a neurologist during their doctor-patient consultation met the inclusion criteria for the pilot study. Ten patients were recruited.

To enable teleconsultation in the GP's consulting room, a technical solution was needed that guaranteed both, a stable data connection for the audiovisual signal and an end-to-end encryption. We relied on HD cameras and large high-resolution screens which guaranteed the best possible image transmission. Technical solutions from the company MEYTEC were used. Next, the premises of the GP practice had to be designed in such a way that the patient and the GP could communicate optimally with each other while being optimally transmitted via video and seeing the screen well.

The focus was on building trust between the patients and the doctors in order to emphasise the "medically accompanying" character of the counselling. The technical base station in the neurology department already existed and had to be newly installed in the GP practice. The GP also coordinated the appointments with the patients and the neurologist. Anamnesis, imaging, laboratory parameters and other technical preliminary examinations were transmitted to the neurologist in advance.

The teleconsultation was always initiated by the GP, followed by a short introduction of all participants. The patients provided information about the epilepsy syndrome and semiology and frequency of the seizures as well as previous and current drug therapies in their own words. The GP added information, e.g. individual observations, family history and suspected diagnoses. The direct questioning of the patients was done by the neurologist, who asked specific questions about the individual disorders and 
the effects of the previous therapeutic measures. In close consultation with the patients, possible further diagnostic steps or changes in medication were discussed. After the joint determination of the further procedure between patient, neurologist and general practitioner, the teleconsultation was concluded. Finally, the patients had the opportunity to ask general questions or questions of understanding.

\section{Recruitment of participants}

Before the intervention the patients gave their written informed consent to participate in the telemedicine consultation. In addition, five of the ten patients agreed to participate in a follow-up interview. Shortly after the intervention, they were contacted by telephone by a female research assistant (MR, diploma pedagogue) with expertise in qualitative social research e.g. in the field of general medicine to make an appointment for a telephone interview.

\section{Characteristics of participants}

The patients of the intervention $(n=10)$ were between 22 and 79 years old. All of them lived in a small town in the rural county Vorpommern-Greifswald in Mecklenburg-Western Pomerania. Six patients had already been given diagnosis of epilepsy, four patients were assessed with a suspected diagnosis during a previous consultation. The participants were able to complete a survey in German, and were considered mentally and physically well, a patient with a mental disability was assisted by his mother who is also his caregiver. The pilot study was performed in a primary care group practice. As a GP and research scientist GF conducted and accompanied the doctor-patient consultations from the perspective of general practice. The hospital-based neurologist FvP is a proven expert in epileptology and head of the Epilepsy Center at the University Medicine Greifswald.

\section{Qualitative data collection}

The qualitative process evaluation of the pilot study was performed by a research associate (MR) of the Institute of General Practice at the University Medical Centre Rostock and took place between January and May 2018. Approximately two weeks after the teleconsultation one-time narrative telephone interviews with a length of five to 30 minutes were conducted with the participating family doctor and the neurologist where there was no previous relationship between the interviewer and the neurologist. Moreover a purposeful sample of five patients based on the ability to participate in an interview was selected. One disabled person was assisted by the caregiver. Otherwise, the patients were interviewed without the presence of third parties and no one dropped out during the course of study. There was no prior relationship between the interviewer and the interviewed patients. In summary, a total of three male and two female patients aged 22 to 64 years with heterogeneous social background were interviewed. Narrative interviews are well-suited to openly elevate individual experiences and to help researchers to understand the personal attitudes and needs of study participants. [11] Initially, the following narrative stimulus was developed and used to stimulate an open narrative in the patients:

"I am interested in your experience with telemedicine care from your GP. Maybe you can just tell me about your last visit to your GP and what was different about it. " 
Following the main narrative, both internal and external questions were asked. Internal questions referred to the main narrative presented and served to deepen the narrated topics. With external questions, new topics were introduced by the interviewer to highlight specific research questions from the interviewee's perspective. In this case external questions related to expectations and concerns about the counselling.

The following stimulus was developed and used for the interviews with the physicians:

We are interested in your experiences with telemedicine care for your patients and what was different than usual.

If not mentioned by the physicians themselves, further external questions referred to previous experiences with telemedicine, advantages from the doctor's perspective, patients benefitting most from telemedicine and continuation of treatment. Finally, demographic data on gender, age and occupation were collected.

The interviews were sound recorded, transcribed verbatim, and pseudonymised. A total of 141 minutes of audio material were recorded. The interviews with the doctors lasted between 23 and 70 minutes and the interviews with the patients between nine and 14 minutes each. Only the physicians took up the offer to view the respective transcripts.

\section{Analysis}

First, right after the interview the interviewer wrote field notes in form of a memo containing both information on the conduct of the interview and on its content. [12] These first memos served as the first analytical step in structuring thoughts, emotions, associations and procedure. Next, data material was analysed and coded on the basis of the content analysis according to Mayring. [13] This was done by a multidisciplinary analysis team consisting of social and health scientists as well as physicians. Initially, the interview material was processed by two coders (MR, GF), then MR, GF, AW, CL and AA contributed to the analysis and interpretation of the data set. By structuring the content of the interviews, it was possible to bundle and differentiate acceptance-promoting attitudes and experiences in relation to telemedical care at the GP from the perspective of all participating groups.

Based on this for each participant group category systems were created by using Microsoft Office. These category systems were developed inductively at the beginning, and were refined several times e.g. after discussions of the working group. When no more additional categories could be found all remaining interviews were included in a category system. This system was thus completed deductively. Our final developed main categories, categories, subcategories and codes are based on the relevant text passages and allow comparison of diverse experiences and evaluations. The results were checked by the participating physicians who also gave feedback on them. Table 1 presents the category system of patients and is divided into four main categories with associated categories and subcategories.

The content analysis of the patient interviews contrasts with the more differentiated category system developed on the basis of the narratives of the physicians. For reasons of clarity, only the five main categories and associated key massages are presented in Table 2. 
Table 1

Category system developed on the basis of the patient interviews

\begin{tabular}{|c|c|c|c|}
\hline Main Category & Category & Subcategory & Codes* \\
\hline \multirow[t]{3}{*}{ Preparation } & \multirow[t]{2}{*}{ Organisational preparation } & $\begin{array}{l}\text { Coordination of the } \\
\text { appointment }\end{array}$ & 1 \\
\hline & & $\begin{array}{l}\text { GP sends findings to } \\
\text { neurologists }\end{array}$ & 2 \\
\hline & Contentwise preparation & Patient education by GP & 4 \\
\hline \multirow{6}{*}{$\begin{array}{l}\text { Expectations } \\
\text { and concerns }\end{array}$} & \multirow[t]{4}{*}{ Hopes and motivation to participate } & Added value by drug expertise & 4 \\
\hline & & GP clarifies uncertainties & 3 \\
\hline & & $\begin{array}{l}\text { More trust in GP than current } \\
\text { neurologists }\end{array}$ & 2 \\
\hline & & Alleviation of drug side effects & 1 \\
\hline & \multirow[t]{2}{*}{ Concerns } & $\begin{array}{l}\text { Data security of video } \\
\text { recording }\end{array}$ & 1 \\
\hline & & Adverse therapy changes & 1 \\
\hline \multirow[t]{9}{*}{ Perception } & Initial experience & Insecurities & 4 \\
\hline & \multirow[t]{3}{*}{ Video and audio technology } & Technical implementation & 7 \\
\hline & & Advantage over phone call & 2 \\
\hline & & Virtual communication & 5 \\
\hline & \multirow[t]{5}{*}{ Doctor-patient conversation } & Trust-building & 3 \\
\hline & & Understandable explanation & 4 \\
\hline & & Thematising prehistory & 3 \\
\hline & & Discussing current medication & 6 \\
\hline & & Reducing fears and insecurity & 3 \\
\hline \multirow[t]{5}{*}{ Acceptance } & \multirow[t]{4}{*}{$\begin{array}{l}\text { Advantage in comparison to the } \\
\text { specialist outpatient clinic }\end{array}$} & $\begin{array}{l}\text { Time and travel savings for } \\
\text { patients }\end{array}$ & 10 \\
\hline & & $\begin{array}{l}\text { Suspected time savings for } \\
\text { doctors }\end{array}$ & 2 \\
\hline & & Cost savings & 1 \\
\hline & & $\begin{array}{l}\text { Organisational relief } \\
\text { (caregivers/ working people) }\end{array}$ & 3 \\
\hline & Assessment of the results & Increased patient safety & 5 \\
\hline
\end{tabular}




\begin{tabular}{|c|c|c|c|}
\hline Main Category & Category & Subcategory & Codes* \\
\hline & & Clarification of further therapy & 8 \\
\hline & & Improved access to specialist & 2 \\
\hline & & $\begin{array}{l}\text { Improved QoL and state of } \\
\text { health }\end{array}$ & 3 \\
\hline & General assessment & Positive evaluation & 7 \\
\hline
\end{tabular}


Table 2

Category system developed on the basis of the physician interviews

Main Category

Previous experiences with audio-visual teleconsultation and outpatient needs

Expectations regarding outpatient telemedicine

Implementation in the practice including preparation, results, and follow-up
Core aspects*

No experiences of the GP

Clinical experiences of the neurologist

Inadequate and uncertain current care situation of the target group

Hopes of the GP for more patient and family safety and improvement of own medical care practice

Neurologist's desire for adequate treatment

GP concerns about mental and social overload of insecure patients and patients in need of care

Neurologist's concern of a higher time expenditure

Sensitive selection of suitable patients

Most preparation effort on the part of the GP

Moderation by GP, inclusion of all parties in case discussion, shared information exchange

Initial diagnosis

planning of further therapy and diagnostic steps

anxiety and stress reducing measures

Documentation and follow-up by GP and neurologist

Facilitated access to specialist care and improved rural supply

Suitable for target group, possible follow up with other patient groups with clinical findings

Positive impact on doctor-patient relationship

More transparent therapy planning for GP and patient

Improved education and level of information for all parties

Reassurance of the GP and family carers

High acceptance by patients and relatives and clarity about positive conditions for acceptance

Positive cost-benefit ratio in terms of time spent and effectiveness from neurologist's perspective 
Main Category

Future challenges and wishes

\section{Core aspects*}

Optimisation of scheduling

Possible increase of the number of patients and diagnoses

Implementation in acute care

Simplified documentation and billability of the specialist consultation

* In contrast to Table 1, the focus here is on the main categories and core aspects derived from the categories and subcategories.

\section{Results}

In the following, we summarise and contrast the results of the content analyses for both patients and physicians.

\section{Expectations and impressions of patients}

Initially, none of the patients had any experience with telemedical consultation and was, thus, informed in advance by the GP about the procedure. The expectations and feelings regarding the upcoming new type of consultation ranged from restlessness, tension, and indifference up to positive expectations of the "video telephony", which was perceived as modern. Overall, the hopes outweighed, for example, by the expected expert opinion regarding the medications taken or regarding suspected epilepsy. The following quotations show that the initial nervousness some patients felt, subsided during the consultation:

"... and I was, at that moment [preliminary talk with the GP] really restless, I have to say. Because I was excited about how this will go on. But this unrest has suddenly disappeared [during the consultation]." (35 years old male patient)

from the very beginning somehow it was strange, that you are sitting in front of the GP but the specialist was also present. This was really weird, but you were pretty fast used to it. That fitted very fast. [...] Well, you didn't really notice that you were practically telephoning over the Internet." (32 years old male patient)

This familiarisation effect to virtual communication was probably also due to the functioning technology and good sound and image quality. The technical solution seemed to recede into the background. Furthermore, the patients reported that a trustful relationship to the mainly unknown specialist could be set up immediately.

However, one women - the oldest interview participant - would have preferred direct contact. 
„... well it is different, isn't it? Face to face contact is always better, but I found it quite okay. The specialist was very kind and you were able to see him really well. All in all, it was good, it is possible to do it this way." (64 years old female care giver)

\section{Final evaluation of patients}

The patients emphasised the amount of time they would normally have to spend visiting the special outpatient clinic. On the one hand, this included the long journey of up to one and a half hour each way, but also the waiting time on site. One patient told us that he also saved on fuel costs and another one was happy for not being forced to put in a day's leave. The mother of the disabled patient in particular was relieved about the significantly reduced organisational effort. Especially multimorbid patients and their caring relatives benefited from telecommunication, even more so if they lived in rural areas.

"...the driving, then I would have had to accompany him [to the specialist], then I would have had to deregister him from the workshop for the disabled and all that. And then I would have had to wait. So, I found it quite good." (64 years old female care giver)

The patients were given a lot of space to tell stories based on the neurologist's inquiries, e.g., about the history of the disease. The experiences with the current medication and possible side effects were perceived as a major topic, with even long-standing therapies were skeptically questioned by the neurologist. This led, for example, to a change in medication. One patient subsequently reported a significantly reduced seizure rate and intensity and therefore an increased quality of life. For some patients, the need for hospitalisation for more in-depth diagnosis which had already been suspected, was confirmed and appointments were made. A patient seemed relieved having found a proper specialist on hand, with the GP also wanting to take over the further coordination of e.g. transport and medication. In addition, a very unsettled patient felt that his concerns were very much taken into account in the consultation and told us in the later interview that the rapid clarification of the suspected diagnosis via video conferencing led to prompt reassurance in the event of further epileptic seizures:

„... the video-conference really helped me. I got calmer, became more balanced. With the next epileptic seizure, I was more relaxed shortly after. (22 years old female patient)

"... it was my first experience with internet telephony. But it is a good thing! It has to be said quite frankly, it is super!" (32 years old male patient)

\section{Preparations and expectations of physicians}

The epileptologist was already experienced in telemedicine and emphasised that the telemedical setting in this pilot study allowed a consultation similar to the epilepsy consultation offered in his clinic.

His main initial concerns referred to the conduct of a proper anamnesis interview as well as to organisational and time efforts. However, especially with reference to the latter, these doubts were removed as he felt the telemedical consultation was well prepared by the GP. In total, he invested on average 15-20 minutes per consultation plus subsequent documentation. He said: 
"The colleague [GP] talks to the patients, prepares them for the fact that now someone from another clinic, whom the patients except for one, I think, do not know personally, will ask very specific questions, that he is in the picture, that he already has medical findings." (epileptologist)

The GP first had to select suitable patients. He identified a need of some of his epilepsy patients to have access to a specialist. It was important to him not to disturb a well-functioning patient-specialist relationship by participating in the teleconsultation with another specialist. However, especially for patients with inadequate medication as well as those with a poor relationship to their neurologist, he saw major advantages of this type of teleconsultation.

In his view, uncertainties in diagnoses and treatment could be clarified rapidly with major advantages for patients. This regarded, for instance, his wish for a second opinion on a patient's unspecific symptoms that seemed to indicate epilepsy.

As to coordination of appointments, the GP experienced an increase in workload. Clearly, the coordination of three parties involved more effort than the coordination of an appointment only between two parties. In particular, the GP was concerned about the mother of the disabled patient, who he perceived to be dominant in discussions. However, during the teleconsultation these concerns turned out to be unnecessary. She got used to this new form of consultation quickly and seemed to be relieved and reassured afterwards. The GPs' positive perception coincides with the statements of the patients. In sum, the GP found the pre- and post-processing work manageable, although he copied the preliminary findings and sent them to the neurologist for preparation. It was an advantage for him that he already knew the patients. Also, the follow-up was not different to conventional consultations, and the additional consultation report from the neurologist finally provided more comprehensive documentation on the patient case.

Nevertheless, for the specialist it was problematic that the patient case could not be saved as a case in the hospital information system and thus could not be billed. Future implementations would have to solve this problem. In addition, due to organisational issues, acute cases and very short-term appointments are a major challenge for this type of teleconsultation. The neurologist saw limited use for patients requiring a detailed neurological examination, e.g. those with Parkinson's disease, other movement disorders or multiple sclerosis. These cases would exceed the time frame and the expertise of the GP, and still require personal face-to-face counselling.

\section{Final evaluation of physicians}

The neurologist emphasised that telemedicine is very well suited for epilepsy patients, as much can be deduced from the medical history. Also, in this view, teleconsultation would also be suitable for patients with other diseases, e.g. those who have already been presented to his or another specialist department, have an up-to-date examination result or a clear treatment plan; patients, in other words, for whom the focus is more on follow-up care. 
"Well, I'm thinking for example of stroke patients, who might at some point have to deal with the question of adjusting blood thinning, adjusting the risk factors, i.e. drugs that influence the risk factors, or patients, who may have been seen by a neurologist with a Parkinson's syndrome and where the point is that if you have an intolerance to the medication, you can perhaps decide to reduce the medication or switch to another one." (epileptologist)

Furthermore, the neurologist described the specialist care in the GP's region as insufficient. It is very difficult for patients to get an appointment at his epilepsy consultation. This is consistent with the experiences of the patients interviewed. The specialist was convinced that the offer of teleconsultation makes it easier to find an appointment quickly with a simple phone call from the GP. In addition, he suspected that some of the study participants would not have visited a specialist outpatient clinic of their own accord. From his point of view, the familiar GP could be a door opener with the offer of teleconsultation and lower the inhibition threshold of hesitant patients.

The GP saw great added value in teleconsultation, especially on a communicative level. In the form carried out here, it would also strengthen the relationship of trust with the patient. Above all, he felt like a partner of the neurologist; in each teleconsultation all three participants had communicated with each other at eye level. He saw himself first and foremost as a moderator who supports patients in describing their complaints and symptoms to the specialist and formulating their concerns. After the consultation, the GP summarised what had been discussed for the patients and discussed how to proceed. In some cases, previously unrecognised problems and gaps in knowledge became apparent. These could be discussed and clarified. In this respect, the teleconsultation offered the possibility to be on the same level of knowledge with the patient and the specialist. This is rarely the case in the German health system, where chronically ill and multimorbid patients consult a multitude of different specialists. The specialist's targeted questions provided the GP with further information about the patients. This was particularly beneficial for the patient-centred treatment of his patients.

GP uncertainties regarding further treatment could also be clarified quickly. Necessary therapy steps or adjustments such as hospital admissions or medication changes could be initiated. According to the neurologist, a brief specialist assessment via the telemedical consultation is a good way of making simple recommendations to the GP and clarifying whether action needs to be taken quickly or not. In case of a larger implementation of teleconsultation - for example, also for other diseases - the GP would like to see a network that takes over the coordination of appointments. In summary, despite the individual challenges both the GP and the neurologist experienced teleconsultation as enriching.

"And I see the added value in the fact that, if you have such a form of access to a specialist, you can choose the time together with the patient and here you can have a much more thorough and more profound form of consultation, if the three of us carry out the whole thing, which is where I see the great benefit and added value in the situation." (GP)

From the neurologist's point of view, the future expansion of this cooperative form of consultation is feasible and worthwhile for all sides involved. In addition, adjustments to the current concept of 
telemedicine should also be considered.

"That's [teleconsultation] something we'll be able to absolutely master if it continues on this scale. It can also be expanded. It's not the mass of patients you see here now and it's not very frequent, regular appointments. Hm and if not, then you just have to think about other concepts, personnel concepts. But the way it works now, it works very well and the decisive thing is that the patients benefit from it. And the second point is that both sides, the general practitioner and us, profit from it. And I think that's also the way we go about it." (epileptologist)

\section{Discussion}

\section{Summary}

Overall, patients accepted the offer of a teleconsultation very well and experienced it as positive. Of course, they first had to get used to the still unfamiliar conditions. However, the trusting environment and the stable relationship with the GP supported this. In addition, with the help of the GP they were able to prepare themselves mentally for the teleconsultation. This further increased acceptance. Both the technical implementation of teleconsultation and the form of communication were perceived by the patients as so self-evident that no criticism was voiced in this regard. Quantitative consequences regarding the therapy regime or further diagnostic measures were not the focus of this feasibility study.

\section{Comparison with existing research}

Previous research shows that patients who use teleconsultation often report some mistrust in data security [14]. In our pilot study, however, only one patient expressed uncertainty about the whereabouts of the video footage. Also, in previous studies patients were skeptical about the outcome of the consultation and possible changes in therapy. This was often related to the fact that patients did not know the specialist beforehand. Our pilot study showed that patients particularly valued the time and cost savings of teleconsultation. Other studies also confirm this [15]. These advantages were also of particular benefit to accompanying relatives of patients in need of care, all the more so if they are employed.

The working group of Salisbury and colleagues investigated so-called private video-first consultation services for primary care in the UK and found high levels of satisfaction especially among young and healthy patients.(16) This satisfaction could be an impetus to include teleconsultation in the GP care portfolio.

Further positive effects for GPs were found in a survey on the implementation of video consultations in general practice that was conducted by Tönnies and colleagues.

Performing a qualitative cross-sectional study, they conducted 15 semi-structured interviews with health policy experts representing different interest groups of the German health system: Health insurance funds, government agencies, professional associations of clinicians, and patient representatives. Using psychiatric specialists as an example, the study showed that stakeholders from health policy perceive 
video consultations as a way to improve primary care. Overall, the interviewees mainly mentioned advantages for GPs in terms of relief through saving time resources and the possibility of referring patients to psychosocial treatment, as well as lower-threshold access on the patient side. [17]

In order to facilitate the implementation of future teleconsultation models for specialists in GP practices, the working group Hoffmann and colleagues developed a checklist with items necessary to successfully implement teleconsultation and make use of specialists in psychiatry as an example. Their checklist corresponds to the findings from our study. Hoffmann et al. summarize that in addition to the concrete clarification of responsibilities and tasks, it is necessary to involve patients in a trusting manner, to structure consultation hours and to promote cooperation between general practitioners and specialists. [18] Especially the latter point is of importance as teleconsultation may increase interprofessional exchange. This enables the joint coordination of diagnosis and therapy.

\section{Strengths and weaknesses}

The present pilot study was conducted in a rural and structurally weak region with a low density of neurologists. There was a clear need for teleconsultation with a neurological expert. The chosen approach of bringing together GP, patient, and specialist in one consultation enables a direct interprofessional exchange, which does not usually take place in this form in the German health care system. At the same time, the chosen way of implementation offers the possibility to optimally integrate the patient's point of view through a patient-centred approach. From the neurologist's perspective, the teleconsultation was comparable in content to the outpatient epilepsy consultation in the hospital. For the patient in particular, there was the possibility to quickly clarify open questions about diagnosis and therapy and to coordinate further steps immediately with the GP and the specialists. Both the GP's perspective and the specialist's perspective could be taken into account.

One weakness of this study is certainly that the GP had the role of both the GP therapist and the study initiator and coordinator. Thus, there was a high intrinsic motivation to optimally conduct the pilot study. This motivation may not be transferable to this extent to a larger-scale study. At the same time, however, the feasibility of the concept was the focus of this pilot study.

Overall, it has been shown that this form of counselling is time-intensive. In a larger-scale study, it should therefore be considered how this time expenditure can be minimised, for example through external appointment coordination.

\section{Implications and need for further research}

The chosen approach offered patients in Germany for the first time the opportunity to consult their GP and a specialist about their epilepsy at the same time. In addition to an improved doctor-patient relationship, this also contributed to patient empowerment, e.g. through possible support of the GP in describing patient-related health problems. Decisions could be made jointly by taking into account the perspective of all three parties. This created a completely new form of doctor-patient contact based on 
partnership. In addition, the targeted preparation of the discussion by the general practitioner helped to implement the exchange with the neurologist in a time-efficient manner.

Basically, the organisation of the consultation was the biggest hurdle: Both the coordination of the patients in the normal consultation hour and the time coordination with the neurologist required an organisational effort. However, the added value of a targeted and comprehensive interprofessional exchange between different disciplines could compensate for the organisational effort of the GP. The actual technical implementation is absolutely mature and was used without any problems throughout the pilot study. The patients also quickly got used to the changed conversation situation.

The optimisation of the organisational processes in the practices and clinics should be the focus of further research work. Following this, a numerically larger feasibility study with accompanying evaluation would be necessary to follow up with an RCT.

\section{Conclusion}

This pilot study provided initial evidence that teleconsultations between patients, specialists and GPs in rural areas are feasible and useful. The use of teleconsultations for selective disease patterns, such as epilepsy in this case, enabled efficient consultations close to the patient's home. Other possible applications could be found, for example, in patients with Parkinson's disease or Guillain-Barré syndrome, who are also limited by immobility. Especially in rural areas with a low density of specialists, this approach can reduce future problems in health care.

Nevertheless, this pilot study only provided evidence for the general feasibility. Findings on long-term efficiency and effectiveness are still lacking and can only be obtained through a larger-scale feasibility study and a RCT. Within the framework of such a study, a uniform appointment management as well as documentation and billing system should also be established. This could minimise preparation time and possibly save time and costs. After all, these factors will be decisive in determining the extent to which specialist teleconsultation can be integrated into the future daily routine of GPs.

\section{Abbreviations}

GP: General practitioner

QoL: Quality of life

\section{Declarations}

\section{Ethics approval and consent to participate}

Ethical approval was obtained from the Ethics committee at Rostock University Medical Center (Ref: A2018-0143) in 2018. All participants involved provided written informed consent. 


\section{Consent for publication}

Not applicable

\section{Availability of data and materials}

The datasets used and/or analysed during the current study are available from the corresponding author on reasonable request.

\section{Competing interests}

The authors declare that they have no competing interests

FvP is on the speakers bureau of Bial, Desitin Arzneimittel, Eisai, GW Pharmaceutical companies, Arvelle Therapeutics, Angelinipharma, Zogenix, and UCB Pharma and reports personal fees and grants from Bial, Desitin Arzneimittel, Eisai GmbH, GW Pharmaceutical companies, Arvelle Therapeutics, Angelinipharma, Zogenix, and UCB Pharma.

\section{Funding}

This pilot study was funded by the Ministry of Economics of Mecklenburg-Vorpommern, Germany, from December 2016 to December 2018. Only the technical material resources to carry out the study were funded. The researchers are independent of the funders.

\section{Author's contributions}

GF and FvP contributed to the design of the work. MR and GF contributed to the design of the work. GF was involved in obtaining the patient appointments. MR and GF were involved in coding the anonymised dataset. MR, GF AW, CL and AA contributed to the analysis and interpretation of the dataset. GF and MR were involved in the writing of the study. MR, AW, CL and AA contributed to the revision of the paper. All authors agreed to the version presented. They agreed to be personally responsible for their own contributions and to ensure that questions about the accuracy or integrity of any part of the paper were adequately addressed.

\section{Acknowledgements}

We would like to thank the Ministry of Economics of Mecklenburg-Vorpommern, Germany, for supporting the project.

\section{References}

1. de Boer, HM. 2010. Epilepsy stigma: Moving from a global problem to global solutions. Seizure 19(10):630-636. https://doi.org/10.1016/j.seizure.2010.10.017 
2. Institute of Medicine (US) Committee on the Public Health Dimensions of the Epilepsies; England MJ, Liverman CT, Schultz AM, et al., editors. Epilepsy Across the Spectrum: Promoting Health and Understanding. Washington (DC): National Academies Press (US); 2012. https://www.ncbi.nlm.nih.gov/books/NBK100604/ Accessed 25 Nov 2021. doi:1017226/13379

3. Hamer HM, Kostev K. Sociodemographic disparities in administration of antiepileptic drugs to adults with epilepsy in Germany: a retrospective, database study of drug prescriptions. CNS Drugs. 2014;28:753-9. doi: 10.1007/s40263-014-0187-x. PMID: 25005594.

4. Wootton, R, Bahaadinbeigy, K, Hailey, D. Estimating travel reduction associated with the use of telemedicine by patients and healthcare professionals: proposal for quantitative synthesis in a systematic review. BMC Health Serv Res. 2011; 11:185. doi:10.1186/1472-6963-11-185

5. Schang L, Kopetsch T, Sundmacher L. Zurückgelegte Wegzeiten in der ambulanten ärztlichen Versorgung in Deutschland [Travel times of patients to ambulatory care physicians in Germany]. Bundesgesundheitsblatt-Gesundheitsforschung-Gesundheitsschutz. 2017;60:1383-1392. German. doi: 10.1007/s00103-017-2643-5.PMID: 29075812.

6. Stentzel U, Piegsa J, Friedrich D et al. Accessibility of general practitioners and selected soecialist physicians by car and by public transport in a rural region of Germany. BMC Health Serv Res 16, 587 (2016). https://doi.org/10.1186/s12913-016-1839-y

7. Keezer MR, Sisodiya SM, Sander JW. Comorbidities of epilepsy: current concepts and future perspective Lancet Neurol.2016;15:106-15. doi:10.1016/S1474-4422(15)00225-2

8. Tong A, Sainsbury P, Craig J. Consolidated criteria for reporting qualitative research (COREQ): a 32item checklist for interviews and focus groups. Int J Qual Heal Care. 2007;19:349-357. doi:10.1093/intqhc/mzm042.

9. AWO Landesverband Mecklenburg-Vorpommern e.V. [ed.]. Aspekte der Armut in MecklenburgVorpommern. Forschungsbericht im Auftrag der Arbeiterwohlfahrt Mecklenburg-Vorpommern. [Aspects of poverty in Mecklenburg-Western Pomerania. Research report on behalf of the Workers' Welfare Association Mecklenburg-Western Pomerania] Schwerin. 2015. German. http://www.awomv.de/armutsstudie.html. Accessed 25 Nov 2021.

10. KV MV Kassenärztliche Vereinigung Mecklenburg-Vorpommern Körperschaft des öffentlichen Rechts [ed.]: Qualität und Sicherstellung in der ambulanten medizinischen Versorgung in M-V. Berichtsjahr 2017 [Quality and assurance in outpatient medical care in Mecklenburg-Western Pomerania.

Reporting year 2017]. 2018. Schwerin. German. https://www.kbv.de/media/sp/KVMV_QB2017.pdf Accessed 25 Nov 2021.

11. Wollny A, Marx G. Qualitative Social Research - Origins and approaches for research in family practice. Part 3: The narrative Interview as a Method of Data Collection. ZfA 09/2010 doi: 10.3238/zfa.2010.0329

12. Rosenthal G. Interpretative Social research: An Introduction. Universitätsverlag Göttingen 2018. https://doi.org/10.17875/gup2018-1103 
13. Mayring, P. Qualitative Content Analysis FQS Forum: Qualitative social research Vol 1, No 2 (2000). http://dx.doi.org/10.17169/fqs-1.2.1089

14. Randhawa RS, Chandan JS, Thomas T, Singh S. An exploration of the attitudes and views of general practitioners on the use of video consultations in a primary healthcare setting: a qualitative pilot study. Prim Health Care Res Dev. 2019;20:e5. doi:10.1017/S1463423618000361

15. van der Velden T, Schalk BWM, Harmsen M, Adriaansens G, Schermer TR, ten Dam MA. Implementation of web-based hospital specialist consultations to improve quality and expediency of general practitioners' care: a feasibility study. BMC Fam Pract. 2019;20:73. doi:10.1186/s12875-0190960-5

16. Salisbury C, Quigley A, Hex N, Aznar C. Private Video Consultation Services and the Future of Primary Care. J Med Internet Res. 2020;22:e19415. doi: 10.2196/19415.

17. Tönnies J, Oeljeklaus L, Wensing M, Hartmann M, Friederich HC, Haun MW. Health policy experts' perspectives on implementing mental health specialist video consultations in routine primary care - a qualitative interview study. BMC Health Serv Res. 2021;21:713. doi: 10.1186/s12913-021-06676-x.

18. Hoffmann M, Hartmann M, Wensing M, Friederich HC, Haun MW. Potential for Integrating Mental Health Specialist Video Consultations in Office-Based Routine Primary Care: Cross-Sectional Qualitative Study Among Family Physicians. J Med Internet Res. 2019;21:e13382. doi: $10.2196 / 13382$.

\section{Supplementary Files}

This is a list of supplementary files associated with this preprint. Click to download.

- COREQReportingChecklist.docx 